\title{
Dynamics of almost strong edge modes in spin chains away from integrability
}

\author{
Daniel J. Yates ${ }^{1}$, Alexander G. Abanov ${ }^{2,3}$, and Aditi Mitra ${ }^{1}$ \\ ${ }^{1}$ Center for Quantum Phenomena, Department of Physics, \\ New York University, 726 Broadway, New York, NY, 10003, USA \\ ${ }^{2}$ Simons Center for Geometry and Physics, Stony Brook, NY 11794, USA \\ ${ }^{3}$ Department of Physics and Astronomy, Stony Brook University, Stony Brook, NY 11794, USA
}

(Dated: December 1, 2020)

\begin{abstract}
Results are presented for the dynamics of an almost strong edge mode which is the quasi-stable Majorana edge mode occurring in non-integrable spin chains. The dynamics of the edge mode is studied using exact diagonalization, and compared with time-evolution with respect to an effective semi-infinite model in Krylov space obtained from the recursion method. The effective Krylov Hamiltonian is found to resemble a spatially inhomogeneous SSH model where the hopping amplitude increases linearly with distance into the bulk, typical of thermalizing systems, but also has a staggered or dimerized structure superimposed on it. The non-perturbatively long lifetime of the edge mode is shown to be due to this staggered structure which diminishes the effectiveness of the linearly growing hopping amplitude. On taking the continuum limit of the Krylov Hamiltonian, the edge mode is found to be equivalent to the quasi-stable mode of a Dirac Hamiltonian on a half line, with a mass which is non-zero over a finite distance, before terminating into a gapless metallic bulk. The analytic estimates are found to be in good agreement with the numerically obtained lifetimes of the edge mode.
\end{abstract}

\section{INTRODUCTION}

Topological systems hosting Majorana zero modes have seen extensive research efforts over the past two decades, both in theory and in experiment, due to their potential to provide nearly error-free quantum computation through the braiding manipulations of nonlocal fermions ${ }^{1-4}$. In particular, spinless superconductors in one dimension (1D) are a potential host for these operators $^{5}$, and there are a growing number of experimental platforms to realize them ${ }^{6-12}$.

However, systems that host topological degrees of freedom at the boundary are often only well understood in the free limit ${ }^{13-16}$, or in some cases the interacting but zero temperature limit ${ }^{17,18}$. At finite temperatures, as one turns on interactions, the edge modes will have finite lifetimes for generic systems. One would expect that temperatures that are small as compared to the single particle topological gap to be the most experimentally relevant. Yet, many intriguing examples are beginning to emerge, where edge modes are stable for long times even at temperatures higher than this bulk single-particle gap $^{19-24}$.

Floquet systems provide new avenues to realize topological edge modes and new topological phases with no static analogs ${ }^{25-32}$. As energy is no longer conserved, and is in-fact being pumped into the system leading to heating ${ }^{33-37}$, infinite temperature studies of the lifetime of edge modes in the context of Floquet systems, are also necessary.

In the face of non-integrable, non-equilibrium dynamics, there are largely two active fields of research that study ways to (somewhat) rein-in ergodicity: many-body localization $^{38}$ and prethermalization ${ }^{39-42}$. This work will focus on clean systems and is therefore unrelated to many-body localization. Additionally, the robustness of the edge modes that will be discussed is not related to prethermalization in the usual sense as the dynamics associated with bulk operators is fully thermalizing. Yet, even with infinite temperatures, moderate interaction strengths, and no disorder, we present situations where edge operators that have an overlap with the topological edge mode in the free limit, can survive for times much longer than bulk thermalization times.

A central object in the study of long lived edge modes has been the concept of the strong zero mode $(\mathrm{SM})^{5,43-45}$ and the almost strong zero mode $(\mathrm{ASM})^{19,20}$. The SM usually can be thought of as many-body generalizations of the Majorana zero modes, however there has been an example of a SM that exists for an integrable-interacting system $^{45}$. The ASM is essentially what becomes of the SM when integrability is broken and it is this quantity whose lifetime is studied ${ }^{20-22,24}$.

Let us note that the existence of a SM is a much stronger statement than the existence of an edge zero mode as the former is a statement for the entire spectrum, not just the ground state. The existence of a SM implies the existence of an edge zero mode, but not vice versa. However whether the edge zero mode is a topological edge mode is a more subtle question. To the best of our knowledge there is no general statement relating the two. However, in some known examples with a SM, the edge zero mode can be interpreted as a topological edge mode after possibly an additional Jordan-Wigner like non-local transformation ${ }^{5}$.

Solving the dynamics of ASMs has only been possible through exact diagonalization (ED) calculations, with the lifetime of the edge operator found to be nonperturbative in the integrability breaking parameter ${ }^{19}$. However, lifetimes extracted numerically are plagued with system size dependencies, and an approach valid in the thermodynamic limit is needed. 
We recently showed a route to estimating the lifetime of $\mathrm{ASMs}^{24}$, utilizing the recursion method ${ }^{46,47}$. This method maps the non-integrable dynamics of the Heisenberg equations of motion for the operator of interest, onto that of a free particle whose dynamics is governed by a 1D, semi-infinite, nearest-neighbor, tight-binding model, also known as the Krylov Hamiltonian. Our previous study $^{24}$ suggested a new interpretation of the slow dynamics of the ASM as the result of the presence of an approximate topological edge state in the fictitious lattice of the recursion method. This approximate edge state is similar to that of a Su-Schrieffer-Heeger $(\mathrm{SSH})^{48,49}$ edge mode, but is only quasi-stationary as the state eventually becomes non-normalizable in the infinite bulk, implying an overlap with bulk states that causes the mode to eventually decay.

This work further builds on our previous work ${ }^{24}$ in the following ways. A toy model is constructed from the numerically obtained parameters of the fictitious lattice, and the edge mode operators of this toy model are discussed. Moreover analytic expressions for the lifetime of the edge mode are derived from the toy model, and compared with the numerically obtained lifetimes.

The paper is organized as follows. In Section II the model is introduced, the SM and ASM are defined, and the recursion method outlined. In Section III, the toy model and some simple variations of it are introduced, and their edge modes discussed. In addition, the parameters of the toy model are explicitly extracted from the numerical data. In Section IV, the continuum limit of the toy model is derived, and an analytic estimate for the lifetime of the ASM is obtained. Following this, in Section V, the toy model is solved, without making the continuum approximation, and an analytic estimate for the lifetime of the ASM is obtained. Comparison between the dynamics from the discrete toy model and the ED dynamics are presented in Section VI, and we present our conclusions in Section VII. Some details are relegated to the appendices.

\section{HAMILTONIAN, STRONG ZERO MODE, ALMOST STRONG ZERO MODE, AND RECURSION METHOD}

We study the $X Y Z$ spin $1 / 2$ chain Hamiltonian with a transverse magnetic field $g$,

$$
\begin{aligned}
H=\sum_{i} & {\left[J\left(\frac{1+\gamma}{2}\right) \sigma_{i}^{x} \sigma_{i+1}^{x}+J\left(\frac{1-\gamma}{2}\right) \sigma_{i}^{y} \sigma_{i+1}^{y}\right.} \\
+ & \left.J_{z} \sigma_{i}^{z} \sigma_{i+1}^{z}+g \sigma_{i}^{z}\right] .
\end{aligned}
$$

We briefly discuss some limiting forms of the above model. For $J_{z}=0$, and after a Jordan-Wigner transformation $^{50,51}$, the model maps to free fermions. For this free case, $\gamma=1$ corresponds to the transverse field Ising model, ${ }^{52}$ and is also equivalent to the Kitaev chain when written in the Majorana representation ${ }^{5}$.
For $J_{z} \neq 0$, and in the Majorana representation, the model corresponds to a chain with nearest-neighbor interactions of strength $J_{z}$, and a superconducting gap of strength $\gamma$. When $J_{z} \neq 0, g=0$ the model is interacting but integrable. In contrast, for $J_{z} \neq 0, g \neq 0$, the system is nonintegrable. We set $J=1$ throughout this paper, and denote the length of the chain by $L$. We will be interested in the nonintegrable case of $J_{z} \neq 0, g \neq 0$.

A convenient starting point is to consider $J_{z}=0, \gamma \neq 0$ when $H$ is similar to the Kitaev chain with a general superconducting gap $\gamma$, and the model hosts topological edge states at the boundary. For these parameters, the system falls under class D of the Altland-Zirnbauer classification scheme ${ }^{15,53,54}$ that is characterized by a discrete $\mathbf{Z}_{2}$ symmetry corresponding to fermion parity. The symmetry is manifest even with interactions. This is evident through the operator

$$
D=\sigma_{1}^{z} \ldots \sigma_{L}^{z}
$$

which commutes with the Hamiltonian (1) for all values of coupling constants.

\section{A. Strong zero mode (SM)}

A key concept that readily generalizes free topological edge states to the interacting case, is that of Strong zero modes $(\mathrm{SM})^{19,20,45}$. A SM is defined as an operator $\Psi$, which commutes with $H$ in the thermodynamic limit, $[H, \Psi] \rightarrow 0, L \rightarrow \infty$, anti-commutes with the global symmetry $\{D, \Psi\}=0$, and is normalizable i.e., $\Psi^{2}=O(1)$. The SM are a statement about the full spectrum of $H$ rather than particularities of the ground state. In particular, the existence of a SM implies that the full spectrum of $H$ is at least doubly degenerate, corresponding to the two different symmetry sectors.

In the free limit, the single-particle topological edge operators are precisely SMs, and in this limit when $g=$ 0 , the SM is trivially $\Psi=\sigma_{1}^{x}$ or, via a Jordan-Wigner transformation, $\Psi=a_{1}$, where $a_{1}$ is the Majorana mode on the first site. In the Ising limit of $g \neq 0, \gamma=1$ the SM has been discussed in Ref. 5 and 45, while for $g \neq 0, \gamma \neq$ 1, the SM was constructed in Ref. 24. The interactingintegrable XYZ model $(g=0)$ was also shown to host a SM localized at the edge of a semi-infinite system ${ }^{45}$. As these operators commute with $H$ in the large systemsize limit, their dynamics is trivial. For small system sizes on the other hand, the SM can decay by tunneling across the wire, acquiring a lifetime that is exponential in system size $\mathrm{s}^{4,55}$. This lifetime can be estimated using perturbative arguments ${ }^{24}$.

\section{B. Almost strong zero mode (ASM)}

As the parameters are tuned away from the integrable point, the operator $\Psi$ will no longer be a SM, with the 
commutation of $H$ failing to drop off as the systemsize is increased en-route to the thermodynamic limit. In this case, where the commutator is very small albeit non-zero in the thermodynamic limit, the operators are called ASMs, and their dynamics is in between that of the trivial dynamics of the exact SM and the featureless dynamics of an infinite temperature non-integrable system $^{47,56-58}$. In particular, the ASM is typically characterized by long lifetimes, despite strong interaction strengths and infinite temperatures ${ }^{20,24}$.

One way to study the dynamics of a (A)SM, which is also particularly well suited to cases where analytic expressions for the SM are unavailable, is to consider the edge auto-correlation function $A_{\infty}$, defined as,

$$
A_{\infty}(t)=\frac{1}{2^{L}} \operatorname{Tr}\left[\sigma_{1}^{x}(t) \sigma_{1}^{x}(0)\right] .
$$

This quantity will act as a good measure of the lifetime of the ASM as long as $\operatorname{Tr}\left[\Psi \sigma_{1}^{x}\right] \sim O(1)$. $A_{\infty}$ measures the lifetime of a Majorana mode to remain on the first site, or equivalently it measures the edge spin coherence as one time-evolves a generic state. The latter is a state that is far from being any exact eigenstate of $H$ and therefore has overlap over a large portion of the spectrum of $H$.

Examples of $A_{\infty}(t)$ for a range of $J_{z}$ are shown in Fig. 1. The lifetime of the ASM is sensitive to small system sizes. In Fig. 1, the lifetime increases as the system size increases. At some point, a large enough system size is reached where the lifetime becomes system size independent, or saturates, allowing ED to be representative of the thermodynamic limit for this quantity. In Fig. 1, this limit is reached for all parameter values.

There is a growing series of studies that predict a lifetime dependence near the Ising limit $\gamma \sim 1$, in terms of the integrability breaking term $J_{z}$, of the form $\sim e^{1 / J_{z}}$ up to logarithmic corrections ${ }^{19,21,22,24}$. This long lifetime can be qualitatively predicted by using prethermal type arguments. In particular, the $O(1)$ energy scale to flip the spin at the boundary is highly off-resonant with the microscopic scale $J_{z}$ that breaks integrability since $J_{z} \ll 1$. Thus, the long lifetime arises from counting the number of microscopic processes $1 / J_{z}$ needed to flip the boundary spin. However as mentioned earlier, the dynamics of bulk operators are fully thermalizing, and this prethermal argument is only valid for the edge operator.

Long lived ASMs have also been demonstrated when the prethermal concept is clearly not applicable, namely when a large energy scale separation is absent in the free case because an external drive in a Floquet setting is not highly off-resonant with the system ${ }^{59}$. Further, long-lived ASM analogs of Floquet $\pi$-modes, which require drives resonant with the single-particle band have been demonstrated even for the clean system with interactions ${ }^{59}$.

A more general perspective to the non-perturbative origin of the lifetime of the ASM, not dependent on prethermal physics, was presented in Ref. 24. Here, the dynamics of the edge operator was mapped to that of a single-particle semi-infinite chain, by means of the recursion method ${ }^{46,47}$. The semi-infinite chain obtained

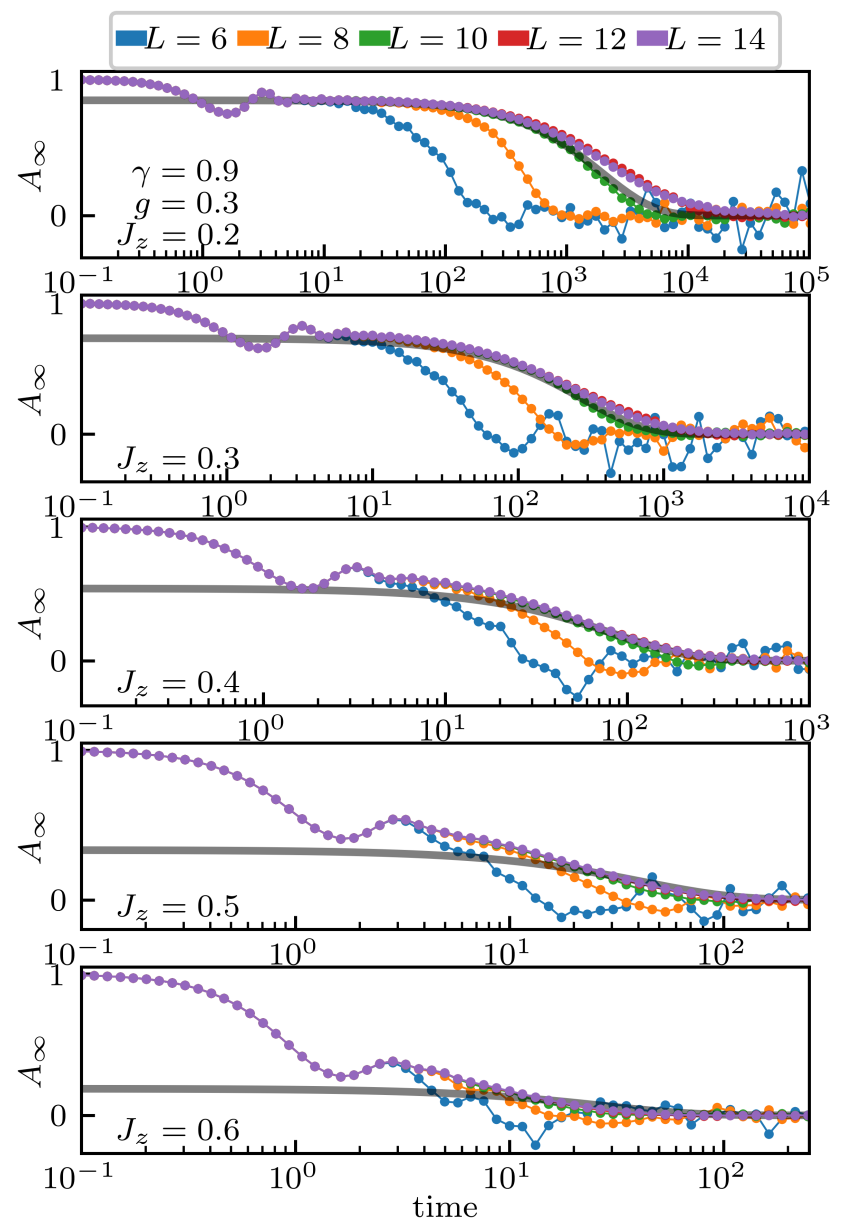

FIG. 1. $A_{\infty}$ for system lengths $L=6,8,10,12,14, \gamma=.9, g=$ .3. From top to the bottom panel $J_{z}=.2, .3, .4, .5, .6$. The dotted data sets are the result of ED calculations. The solid black curve is the result of approximating $A_{\infty}$ by Eq. (45), with $N=400$, where $b_{n}$ used in this computation, are generated by the Lanczos algorithm Eq. (7), for $L=12$, and are shown in Fig. 2. Note the different ranges on the $x$-axes.

this way was found to have topological features similar to that of the SSH model, and therefore this mapping allowed one to show that the slow dynamics of the ASM was a result of a long lived edge mode of a generalized or perturbed SSH model.

The goal of the current paper is to further build on the results of Ref. 24. A more detailed numerical study of the dynamics of the ASM will be supplemented by analytic estimates for the lifetime. The latter will be obtained by exploiting the mapping to a free system to construct suitable toy models. The quasi-stable edge modes of the toy model will be solved for. 


\section{Lanczos algorithm and the Krylov Hamiltonian}

The time evolution of an operator in a generic integrable or non-integrable system can be mapped to single particle dynamics on a semi-infinite chain ${ }^{46}$. In this section we outline this method. The exponential complexity of solving the dynamics enters into the calculation of the hopping parameters on this chain which we denote by $b_{n}$.

The Heisenberg time-evolution operator can be written as

$$
e^{i H t} O e^{-i H t}=\sum_{n=0}^{\infty} \frac{(i t)^{n}}{n !} \mathcal{L}^{n} O,
$$

where we define

$$
\mathcal{L} O=[H, O]
$$

To employ the Lanczos algorithm, we will recast operator dynamics into vector dynamics by defining $\mid O)=O$. Since we are concerned with infinite temperature quantities, we have an unambiguous choice for an inner product on the level of the operators,

$$
(A \mid B)=\frac{1}{2^{L}} \operatorname{Tr}\left[A^{\dagger} B\right] .
$$

The Lanczos algorithm iteratively finds the operator basis that tri-diagonalizes $\mathcal{L}$. We begin with the seed "state", $\left.\mid O_{1}\right)$, and let $\left.\left.\mathcal{L} \mid O_{1}\right)=b_{1} \mid O_{2}\right)$, where $b_{1}=$ $\sqrt{\left.|\mathcal{L}| O_{1}\right)\left.\right|^{2}}$. The recursive definition for the basis operators $\left.\mid O_{n \geq 2}\right)$ is,

$$
\left.\left.\left.\mathcal{L} \mid O_{n}\right)=b_{n} \mid O_{n+1}\right)+b_{n-1} \mid O_{n-1}\right),
$$

where we define $b_{n}=\sqrt{\left.|\mathcal{L}| O_{n}\right)\left.\right|^{2}}$. It is straightforward algebra to check that the above procedure will iteratively find basis operators that yield a $\mathcal{L}$ which is tri-diagonal, and of the following form,

$$
\mathcal{L}=\left(\begin{array}{cccc} 
& b_{1} & & \\
b_{1} & & b_{2} & \\
& b_{2} & & \ddots \\
& & \ddots &
\end{array}\right) .
$$

This basis spanned by $\left.\mid O_{n}\right)$ lies within the Krylov subspace of $\mathcal{L}$ and $\left.\mid O_{1}\right)$. We refer to this tri-diagonal matrix as the Krylov Hamiltonian.

An important aspect of this technique, often overlooked when discussing chaos, is that the values of $b_{n}$ are highly dependent on the choice of seed operator. Further, outside of special cases, namely a Hamiltonian that is free, the exact solution to the operation $\left.\mathcal{L} \mid O_{n}\right)$ will require ED, or similar methods with equivalent costs. This method does not escape the rapidly growing exponential wall of complexity. In cases where the calculation of all $b_{n}$ are possible, the above algorithm will return a value of $b_{\text {end }}=0$. Knowledge of the full set of $b_{n}$ results in full knowledge of the dynamics of the seed operator.
For free systems, the operation $\mathcal{L} \mid O_{n}$ ) can be efficiently solved when in the Majorana basis. If the starting operator is a single Majorana then the dimension of the Krylov-subspace of that operator will scale as $2 L$, as free system dynamics can only mix the individual Majoranas among themselves.

Outside of free problems, the size of the full set of $\left.\mid O_{n}\right)$ will be large. For example, a system size of $L$ will have $\sim$ $2^{2 L}$ possible basis operators. For all intents and purposes we treat $\mathcal{L}$ as a semi-infinite chain. If the number of solved $b_{n}$ is insufficient for the quantity of interest, the typical approach is to supplement the known set with approximate $b_{n}$ that are calculated based off of trends established among the known hoppings.

Starting with the seed state $\left.\left|O_{1}\right|=\mid \sigma_{1}^{x}\right)$, we can recast $A_{\infty}$ into an equivalent form,

$$
A_{\infty}(t)=\left(e^{i \mathcal{L} t}\right)_{1,1}
$$

Now, following the above discussion, the dynamics of $A_{\infty}$ has been transformed into that of a semi-infinite singleparticle problem. The details of the semi-infinite chain will be discussed in subsequent sections. Ref. 24 showed that the slow dynamics of $A_{\infty}$ is a result of topological modes residing at the left boundary (origin) of the Krylov wire. Ref. 24 also showed how the parameters of the Krylov wire change for different choices of edge operators such as $\left.\left.\mid O_{1}\right)=\mid \sigma_{1}^{y, z}\right)$.

\section{GENERAL STRUCTURE OF $b_{n}$ AND THE TOY MODEL.}

In this section we will discuss the general structure of the Krylov wire in Eq. (8) parameterized by the hoppings $b_{n}$, and use our observations to motivate some toy models.

\section{A. Discussion of $b_{n}$}

Fig. 2 shows the first $400 b_{n}$ calculated for the same parameters as those in Fig. 1. There are three main aspects to the $b_{n}$, the roughly linear ramp for small $n$, the staggering or dimerization of the $b_{n}$ superimposed on the ramp, and the system size dependent "plateau" or flat region after the linear ramp ends. Note that we will use the words staggering and dimerization interchangeably to denote the even-odd pattern of the $b_{n}$ typical of an SSH model ${ }^{48,49}$.

The linear ramp is expected for non-integrable systems ${ }^{47}$, with the slope increasing with $J_{z}{ }^{24}$. In contrast, the staggering becomes more pronounced as $J_{z}$ is decreased, and coincides with the longer-lived $\mathrm{ASM}^{24}$. The $b_{n}$ resemble an SSH model with a sign of the dimerization which is topological, but with a linear slope superimposed on the hoppings. We expand on these points further when we develop the toy model. 
Fig. 2 also shows that the height of the plateau does not experience a saturation in system size, $L$, even though $A_{\infty}$ in Fig. 1 does saturate with system size. This implies that the lifetime of $A_{\infty}$ must be independent of plateau heights, and rather, must be primarily dependent on the nature of staggering and the linear ramp.

As emphasized earlier, many features depend on the initial seed operator. Thus, while an overall slope is expected for all operators in a non-integrable model, the nature of the dimerization, such as its sign, and how long it persists into the bulk, will depend on whether the operator has strong overlap with the ASM or whether an operator has little overlap. For the latter, the autocorrelation function will have a short lifetime ${ }^{24}$.

One can supplement the naive Lanczos algorithm in Eq. (7) with an additional step of Gram-Schmidt orthogonalization of the newly produced $\left.\mid O_{n}\right)$, against $\left|O_{n^{\prime}}\right|$ for all $n^{\prime}<n$. This ensures orthogonality and is necessary for discussing the details of $b_{n \gtrsim 100}$. A comparison between the Lanczos algorithm and the Gram-Schmidt orthogonalization is presented in Appendix A. However it is helpful to note here that since the error between the two numerical schemes is apparent only at large $n$, for the times shown in Fig. 1, the approximate $A_{\infty}$ (discussed later, c.f. Eq. (45)) sees minor changes when the GramSchmidt is implemented. Moreover, the Gram-Schmidt orthogonalization requires too much computer resources to be performed for $L=14$, and hence the corresponding data is absent in this paper.

The two different data sets, one from the Lanczos algorithm, and the other from the Gram-Schmidt orthogonalization, for accessible $L$ and different $J_{z}$, are shown in the top row of Fig. 2. As mentioned above, the two data sets have perfect agreement for $n<100$ and qualitative agreement for the entire domain. Outside of Fig. 1 and Fig. 2, we will concentrate on the Gram-Schmidt orthogonalized data. Although this data set is available only up to $L=12$, Fig. 1 shows that $L=12$ is sufficient to attain saturated i.e, system size independent lifetimes for all shown $J_{z}$.

\section{B. Calculation of edge states of the Krylov lattice}

The $b_{n}$ s in Fig. 2 suggest a topological SSH model with a linear slope. Therefore the topological edge states of this generalized SSH model become a likely candidate for the origin of the slow dynamics of the ASM. Motivated by this, we search for the form of the edge states for generalized SSH models. The calculation is the same as in a regular $\mathrm{SSH}$ model with no slope.

We solve the eigenvalue equation $\mathcal{L}|\psi\rangle=E|\psi\rangle$, which due to the tri-diagonal form of $\mathcal{L}$ in Eq. (8) can be rewritten into an iterative transfer matrix form,

$$
\left(\begin{array}{c}
\psi_{l+1} \\
\psi_{l+2}
\end{array}\right)=\left(\begin{array}{cc}
0 & 1 \\
-\frac{b_{l}}{b_{l+1}} & \frac{E}{b_{l+1}}
\end{array}\right)\left(\begin{array}{c}
\psi_{l} \\
\psi_{l+1}
\end{array}\right)
$$

Defining, $\phi_{2 l-1}=\psi_{2 l-1}$ and $\eta_{2 l}=\psi_{2 l}$, at $E=0$ we have,

$$
\begin{aligned}
\phi_{2 l+1} & =-\frac{b_{2 l-1}}{b_{2 l}} \phi_{2 l-1}, \\
\eta_{2 l+2} & =-\frac{b_{2 l}}{b_{2 l+1}} \eta_{2 l} .
\end{aligned}
$$

Thus the even sites and odd sites decouple for $E=0$. The modes with support on the odd (even) sites can be calculated by substituting $\psi_{1(2)}=1$ into the above equation.

As a point of orientation, note that for an ideal semiinfinite SSH model extending from the origin to the right, $\phi$ is normalizable and corresponds to the left boundary mode when in the topological phase. For this case, $b_{1} / b_{2}=b_{2 n-1} / b_{2 n}$, and the normalized $\phi$ is found to be ,

$$
\phi_{2 l-1}=\sqrt{1-r^{2}} r^{l-1} ; \quad r=\frac{b_{1}}{b_{2}} .
$$

Eq. (10) is completely general and $\phi, \eta$ can also be constructed for the numerically obtained hoppings $b_{n}$ shown in Fig. 2. The corresponding $|\phi|^{2}$ are plotted in the lower panels. Outside of free cases $\left(J_{z}=0\right)$, or integrable cases $(g=0)$, the generic behavior of the $b_{n}$, as shown in Fig. 2, will not host an exact edge state at the origin, but rather only an approximate one. This implies that $\left|\phi_{l}\right|^{2}$ will appear as a localized edge mode for small $l$, but for large $l$ will not be decaying sufficiently to yield a normalizable state.

The lower panels of Fig. 2 show that the decay of $\left|\phi_{l}\right|^{2}$ is strongest at small $l$, corresponding to where the staggering in the $b_{n}$ is the strongest. Additionally, this initial decay is more rapid for smaller $J_{z}$, which also coincides with $b_{n}$ that stagger with greater amplitude in comparison to the $b_{n}$ for larger $J_{z}$. Moreover, the $b_{n}$ for smaller $J_{z}$ also stagger for a longer range of $n$, i.e, the staggering continues further into the bulk. As a result, for small $J_{z}$, non-trivial staggering of the $b_{n}$ occurs beyond the end of the ramp, as opposed to that for larger $J_{z}$. This is evident in the appearance of minima for $\left|\phi_{l}\right|^{2}$ at small and intermediate $n$, for larger values of $J_{z}$, indicating poor normalization of the mode. In contrast, there is a lack of a well defined minima for $\left|\phi_{l}\right|^{2}$ for smaller $J_{z}$, and thus a behavior more akin to a SM.

We also note the differences between the $b_{n}$ obtained from the two different numerical orthogonalization schemes in the top panels in Fig. 2. These differences do not affect the magnitude or location of the minima of $\left|\phi_{l}\right|^{2}$ shown in the lower panels.

\section{Generalized SSH models}

The Heisenberg dynamics of $\sigma_{1}^{x}$ according to Eq. (1) generate $b_{n}$ that contain many details. However here we introduce a series of toy models that address the main features of the $b_{n}$ addressed in Sec. III A. 


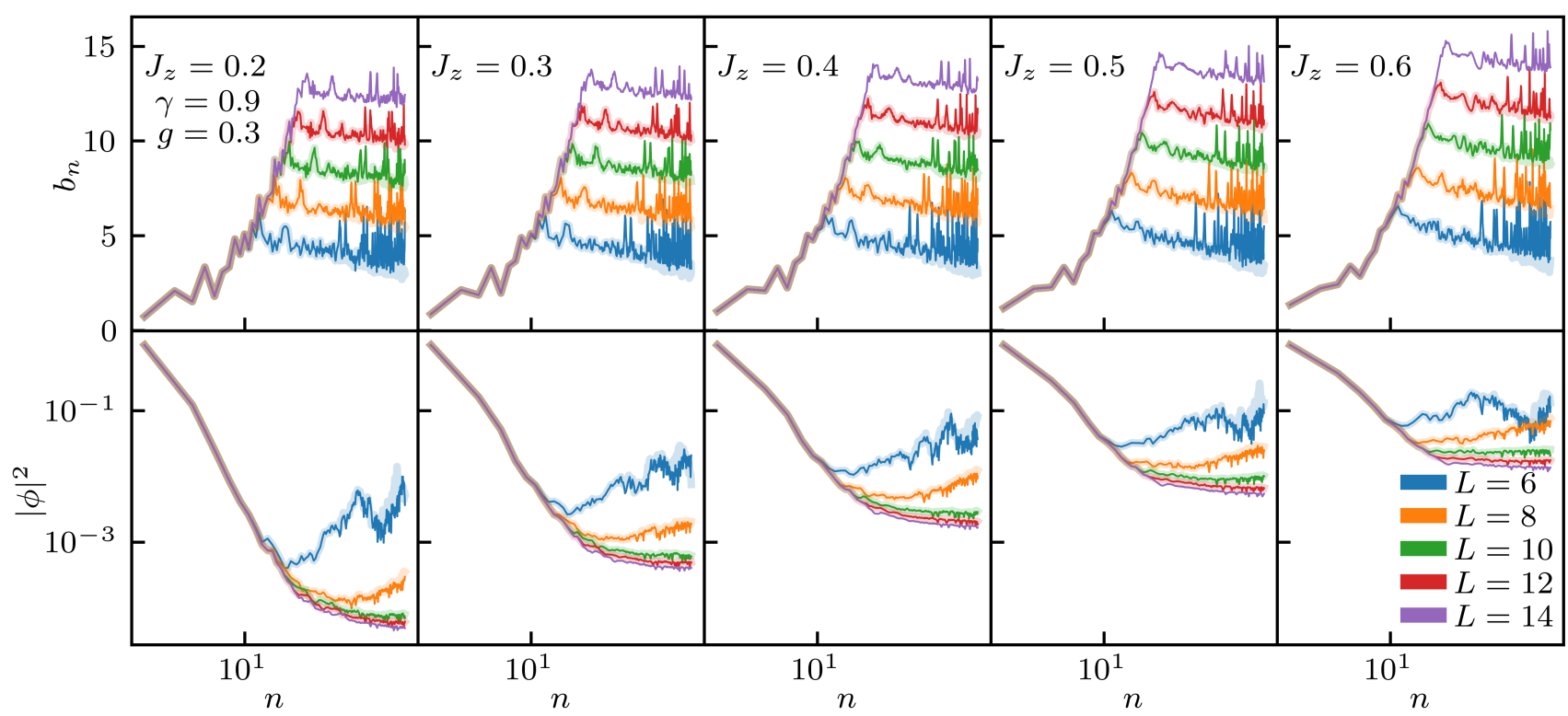

FIG. 2. Top panel: $b_{n}$ plotted for system lengths $L=6,8,10,12,14, \gamma=.9, g=.3$. Left to right, $J_{z}=.2, .3, .4, .5, .6$. The sharp, thin data-sets correspond to the Lanczos algorithm, Eq. (7). The translucent, thick data-sets for $L=6$ - 12 , correspond to the Gram-Schmidt orthogonalized Lanczos algorithm discussed in Appendix A. Bottom: The mod-square of the wave-function $|\phi|^{2}$ of the edge state of the Krylov lattice plotted for the same parameters. The thin, sharp data set corresponds to calculating $\phi$ for the thin sharp $b_{n}$ of the top row, with analogous statements for the translucent, thick data set. The $\phi$ are not normalized, with $\phi_{1}=1$ for all data sets.

The first model we consider is a nearest neighbor tightbinding model with linear slopes for the hopping parameters, and with even and odd site hopping parameters having different slopes,

$$
b_{n}= \begin{cases}\alpha_{1} n+\delta_{1} & n \text { odd } \\ \alpha_{2} n+\delta_{2} & n \text { even }\end{cases}
$$

An example of this model is plotted in the upper left panel of Fig. 3. We choose $\alpha_{1}>\alpha_{2}$ and $\delta_{1}<\delta_{2}$ because this choice ensures that the even and odd hoppings intersect for positive $n$. Moreover, in the $\alpha_{1,2} \ll 1$ limit, the region to the left of the intersection is topologically non-trivial, with the intersection of the slopes equivalent to a topological phase transition. Consistent with this view, the edge mode calculations for $\phi$ and $\eta$ respectively produce localized states at the left boundary and at the topological transition. This is shown in the lower left panel of Fig. 3 where the topological transition occurs around $n \sim 20$. This figure also shows that non-zero slopes $\alpha_{1,2}$ do not remove the edge-modes of the $\mathrm{SSH}$ model entirely.

Now that we have shown that the zero mode considerations of the SSH model are relevant, we move onto a second model, more aligned with the ED data, in the thermodynamic limit. In particular we consider $b_{n}$ that are such that the staggering falls off to zero in the bulk, so that the bulk of the system can be thought of as a

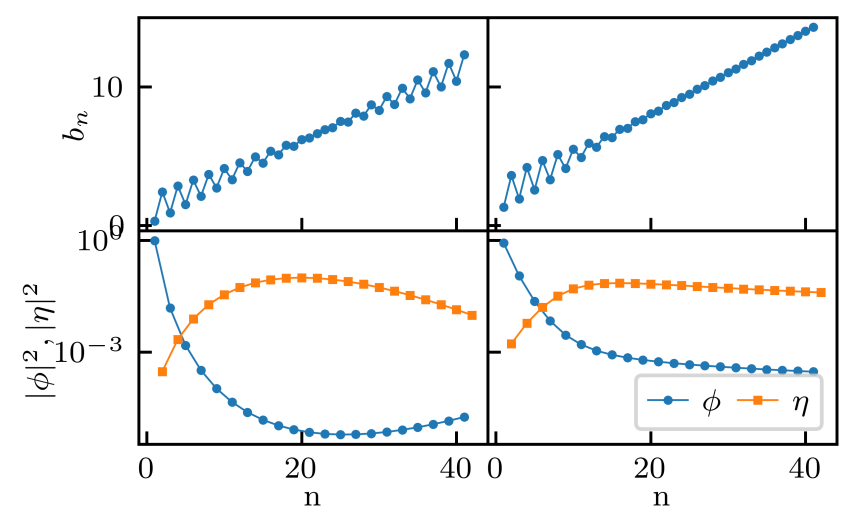

FIG. 3. Demonstration of zero modes within the Krylov Hamiltonian. Top row: $b_{n}$ for the toy model Eq. (13) on the left, and the toy model Eq. (14) on the right. Bottom row: the corresponding $|\phi|^{2},|\eta|^{2}$ for the above $b_{n}$.

metal. This model is,

$$
\begin{aligned}
b_{n} & =h_{n}+(-1)^{n} \tilde{h}_{n}, \\
h_{n} & =\alpha n+\delta, \\
\tilde{h}_{n} & =\frac{M_{0}}{2\left[\left(\frac{n}{n_{0}}\right)^{\beta}+1\right]} .
\end{aligned}
$$

Above, $M_{0}$ will have the interpretation of a mass, and we 
will show this explicitly when we derive the continuum limit of the model.

An example of the above toy model is shown in the right column of Fig. 3 . The staggering of the $b_{n}$ starts off with a maximum value of $M_{0}=2$ at the edge, and extends over a region of width $n_{0} \sim 10$, before decaying to zero. The length scale over which the staggering decays is $1 / \beta=.25$. A linearly growing hopping of rate $\alpha=.3$ is superimposed on all the $b_{n}$ and we take $\delta=2$. We see in the bottom right panel of Fig. 3 that the $\phi$ zero mode remains (approximately) localized, while the $\eta$ zero mode is no longer localized. This is reminiscent of a topological phase transition where the edge mode becomes delocalized when the phase is critical. There is some decay for both $\phi$ and $\eta$, well beyond $n_{0}$ which is slower than $1 / n$, and indicates that there are no longer any zero modes as the wavefunctions are not normalizable.

The model in Eq. (14), after the mass has decayed, assumes linearly growing $b_{n}$, i.e, a ramp that continues without interruption. For a system of finite length however, this ramp will eventually terminate into a plateau as seen in Fig. 2. In order to compare with the numerical simulations of finite length chains, we will consider $b_{n}$ which will follow Eq. (14) up to a certain distance, after which it will saturate and form a "plateau" 57 . As we see saturation in the lifetime of the $A_{\infty}$, we expect that as long as this plateau onset occurs after the decay of the staggering, it should not affect the lifetimes. This issue is also discussed in detail in Appendix E.

Due to these considerations, we introduce another toy model, a modification of Eq. (14),

$$
b_{n}=\left\{\begin{array}{ll}
h_{n}+(-1)^{n} \tilde{h}_{n} & n \leq M \\
h_{M} & n>M
\end{array},\right.
$$

where we take the point $M$ at which the ramp ends and the plateau begins to be $M \gg n_{0}$. This toy model gives the same physics as the model in Eq. (14) at small $n$, but at $n=M$, the $b_{n}$ saturate in value, mimicking a metal. This assumption of a perfect flat metal for $h_{n>M}$ will serve as a good first order model.

\section{Comparison between the exact $b_{n}$ and the toy model}

We now demonstrate that the above models will capture the essential features of the exact $b_{n}$ obtained from the ED computation of the Heisenberg dynamics of $\sigma_{1}^{x}$.

With the aim of capturing the underlying trends proposed in Eq. (14) for small $n$, we consider neighboring averages and differences as a simple numerical estimate of the parameters,

$$
\begin{aligned}
& h_{n} \sim \frac{b_{n}+b_{n+1}}{2}, \\
& \tilde{h}_{n} \sim(-1)^{n}\left(\frac{b_{n}-b_{n+1}}{2}\right) .
\end{aligned}
$$

The values for $h, \tilde{h}$ are plotted in Fig. 4 . Additionally, the best fit of the first twenty sites of $h_{n}$ is used to extract the values of $\alpha, \delta$. While there is some small variability between the different values of $J_{z}$, the best fit line appears to accurately depict the $h_{n}$ values.

The $\tilde{h}_{n}$ plot is more complicated as it has a noise whose amplitude is on the order of $M_{0}$. A seven site moving average allows for the trend of the mass to be better visualized. With the averaging, the trend of $\tilde{h}_{n}$ does qualitatively agree with the model of Eq. (14c). When $\beta \gg 1$, the length scale over which the mass drops to zero becomes smaller, and Eq. (14c) approaches a square wave function. A fit of the $\tilde{h}_{n}$ to a square wave is also shown in the lower panel of Fig. 4.

\section{LIFETIME ESTIMATE FROM THE CONTINUUM MODEL}

We plan to derive an analytic estimate for the lifetime of the ASM of the toy model in Eq. (14). For this it is convenient to derive a continuum version of this model. We outline the derivation below with details relegated to Appendix B.

\section{A. Continuum model}

Let us write the toy model quite generally as the following nearest-neighbor hopping Hamiltonian,

$$
H_{K}=\sum_{n} b_{n}\left(c_{n}^{\dagger} c_{n+1}+c_{n+1}^{\dagger} c_{n}\right)
$$

The corresponding Schrodinger equation takes the form,

$$
i \partial_{t} \Psi_{n}=b_{n} \Psi_{n+1}+b_{n-1} \Psi_{n-1} .
$$

To transform to the continuum limit we assume that the hopping parameters and the wavefunction can be written as

$$
\begin{aligned}
\Psi_{n} & =i^{n}\left[\psi_{n}+(-1)^{n} \tilde{\psi}_{n}\right], \\
b_{n} & =h_{n}+(-1)^{n} \tilde{h}_{n},
\end{aligned}
$$

where $\psi_{n}, \tilde{\psi}_{n}, h_{n}, \tilde{h}_{n}$ are all assumed to be smooth, slowly varying functions of $n$.

Measuring distance in lattice spacings $x=n$, introducing continuous notations $\psi_{n}=\psi(x)$ etc., and in terms of the spinor,

$$
\tilde{\Psi}=\left(\begin{array}{c}
\psi(x) \\
\tilde{\psi}(x)
\end{array}\right),
$$

the continuum limit of Eq. (19) becomes (see Appendix B for details),

$$
i \partial_{t} \tilde{\Psi}=\left[\sigma^{y} m(x)+\sigma^{z}\left\{i \partial_{x}, h(x)\right\}\right] \tilde{\Psi}
$$




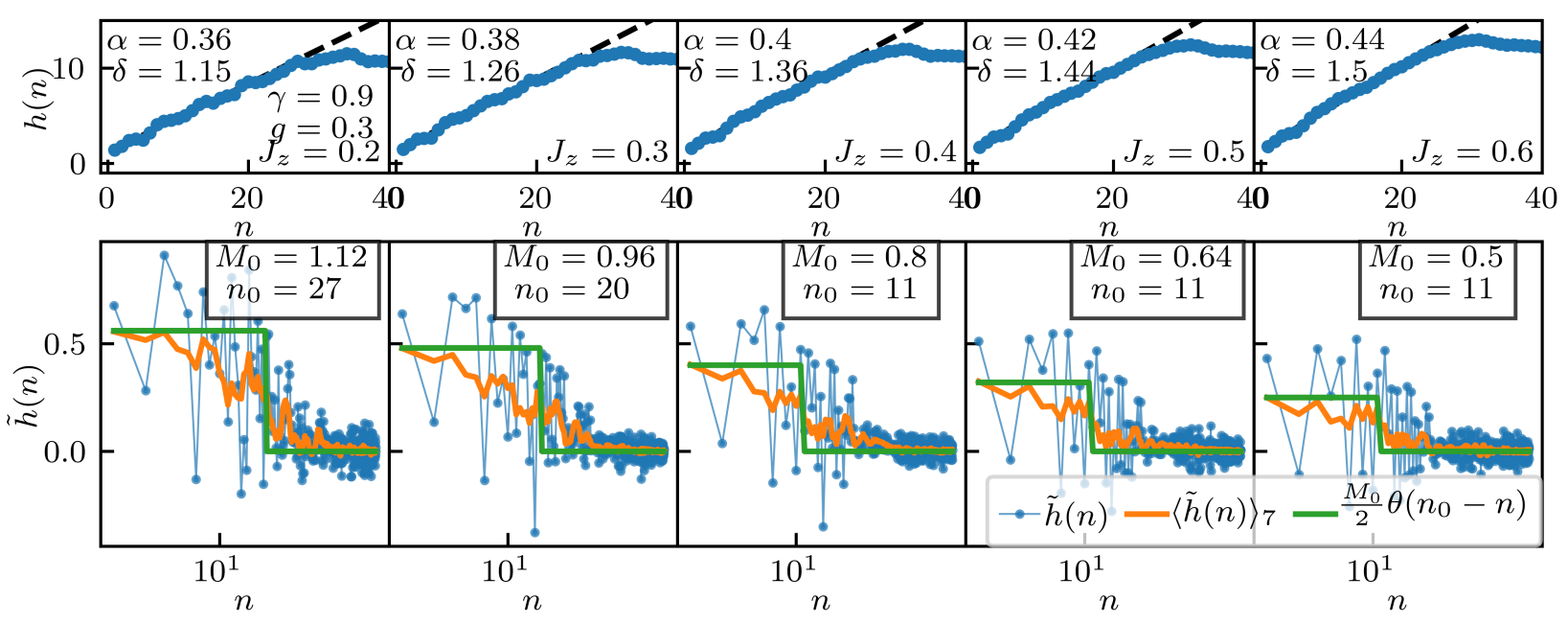

FIG. 4. $h_{n}, \tilde{h}_{n}$ plotted for $L=12$ with $g=.3, \gamma=.9$ and several $J_{z}$, with numerical values determined for parameters $\alpha, \delta, M_{0}, n_{0}$. Top row: $h_{n}$ plotted along with a best fit of the linear ramp. Bottom row: $\tilde{h}_{n}$ plotted along with its moving 7 -site average, $\left\langle\tilde{h}_{n}\right\rangle_{7}$, and with a square wave approximating the moving average $\theta\left(n_{0}-n\right) M_{0} / 2$. $2 M_{0}$ is set as $\left\langle\tilde{h}_{1}\right\rangle_{7}$ and $n_{0}$ is determined starting at the far right $n$ and stepping backwards until $\left\langle\tilde{h}_{n}\right\rangle_{7}$ grows larger than $M_{0}$.

Above the mass is defined as,

$$
m(x)=2 \tilde{h}(x)-\partial_{x} \tilde{h}(x) .
$$

One can easily bring the Dirac equation (23) to the conventional form using reparametrization of space coordinate. Namely, changing variables $x \rightarrow X(x)$ so that

$$
\begin{aligned}
& X=\int_{0}^{x} \frac{d x^{\prime}}{2 h\left(x^{\prime}\right)}, \\
& \tilde{\Psi}=\frac{1}{\sqrt{h}} \chi,
\end{aligned}
$$

we transform (23) to

$$
i \partial_{t} \chi=\left[\sigma^{z} i \partial_{X}+\sigma^{y} m(X)\right] \chi,
$$

where

$$
m(X)=2 \tilde{h}(X)-\frac{\partial_{X} \tilde{h}(X)}{2 h(X)} \approx 2 \tilde{h}(X) .
$$

In the last approximation we assumed that $\tilde{h}$ changes only slowly in space which is consistent with the lattice toy model (14).

It is straightforward to construct a potential zero mode solution,

$$
\chi(X)=e^{\sigma^{x} \int_{0}^{X} m(Y) d Y} \chi_{0},
$$

where $\chi_{0}$ is a constant spinor. The above is a timeindependent solution of the Schrodinger equation (23). It corresponds to a zero energy solution, and is a zeromode or SM if normalizable.

We note the boundary condition of $\Psi(0)=0$, translates into $\sigma^{x} \chi(0)=-\chi(0)$ and ultimately into $\chi_{0} \sim$
$(1,-1)^{T}$, and so it is clear that the necessary condition for the normalization of (29) is the condition $\int^{X} m(Y) d Y \rightarrow+\infty$ as $X \rightarrow+\infty$. In particular the normalizability condition is satisfied for $m(X) \rightarrow m>0$ and in this case Eq. (29) is a true zero-mode (or SM) solution of the Dirac equation (23) on a half-line.

\section{B. Connecting toy model parameters with the continuum model}

Rewriting the toy model expression (14b) as $h(x)=$ $\alpha x+\delta$ and substituting it into (25) we obtain

$$
x=\frac{\delta}{\alpha}\left(e^{2 \alpha X}-1\right) .
$$

We can now proceed and express all parameters given by the toy model (14) in continuum notations as functions of the transformed coordinate $X$

$$
\begin{aligned}
h(X) & =\delta e^{2 \alpha X}, \\
\tilde{h}(X) & =\frac{M_{0}}{2\left[\left(\frac{e^{2 \alpha X}-1}{e^{2 \alpha X}-1}\right)^{\beta}+1\right]}, \\
& \sim \frac{M_{0}}{2\left[e^{2 \beta \alpha\left(X-X_{0}\right)}+1\right]}, \\
\Rightarrow m(X) & \sim \frac{M_{0}}{e^{2 \beta \alpha\left(X-X_{0}\right)}+1} .
\end{aligned}
$$

We observe that in the toy model (14) the Dirac mass persists for some range $x<x_{0}$ and then decays to zero. In the space corresponding to the transformed coordinate $X$ the length of the finite mass region is contracted exponentially. This makes sense as the wavefunction/particle 
sees constantly increasing hopping strengths as it progresses further into the bulk.

The equation (27) corresponds to a Dirac Hamiltonian with a mass that is non-zero up to a certain distance $\sim X_{0}$, beyond which the Hamiltonian is gapless corresponding to that of a metallic lead.

\section{Decay rate in the continuum model}

Eq. (34) suggests that for large enough $\beta$, we may replace the spatial dependence of the mass (34) by the step function

$$
m(X) \approx M_{0} \theta\left(X_{0}-X\right) .
$$

The spectrum of the model (27) on a half-line $X \geq 0$ with the mass (35) is continuous. It has a quasi-stable mode corresponding to an approximate zero mode (29). The decay rate of this mode can be found straightforwardly (see Appendix C for details). It is given by

$$
\Gamma_{A} \sim 4 M_{0} e^{-2 M_{0} X_{0}} .
$$

The exponent can be clearly seen from the semi-classical expression for tunneling amplitude $\exp \left(\left.i \int_{0}^{X_{0}} \sqrt{E^{2}-M_{0}^{2}} d X\right|_{E=0}\right)=e^{-M_{0} X_{0}}$, corresponding to the tunneling probability (36). Expressing (36) in terms of the original coordinate using (30) we obtain

$$
\Gamma_{A} \sim 4 M_{0} e^{-\frac{M_{0}}{\alpha} \log \left(\frac{\alpha x_{0}}{\delta}\right)} .
$$

The decay rate (37) is exponentially small in the size of the staggering represented by $M_{0}$ in the continuum model. This explains why the decay rate of the boundary spin is strongly suppressed by the staggering in the SSH model in Krylov space. Both the result (37) and its derivation are very intuitive in the continuum model (see Appendix C). However, for the values of the parameters obtained from the spin chain (see Fig. 4) the continuum limit of the Krylov Hamiltonian is not fully justified. This is why in the next section we derive the decay rate directly in the discrete model without appealing to additional assumptions necessary for the continuum limit to hold.

\section{LIFETIME ESTIMATE FROM THE DISCRETE TOY MODEL}

We now calculate the lifetime of the ASM within the discrete setting of the Krylov Hamiltonian. To this end, we consider a finite Hamiltonian of length $N, H_{N}$, and connect it to a metallic semi-infinite bulk.

In particular, the Hamiltonian $H_{N}$ is a tight-binding model with $N$ sites, and nearest neighbor hopping $u_{i}$ and no onsite potential. We connect the right end of $H_{N}$ to the semi-infinite metal, where the latter is modeled as a tight-binding model with uniform hoppings. We are interested in the Green's function on the first site, which we denote as the surface Green's function $G_{S}^{(N)}(E)$. Its explicit form is, (see Appendix D),

$$
G_{S}^{(N)}(E)=\left[\left(E-H_{N}-\hat{\Sigma}\right)^{-1}\right]_{1,1}=\left(\begin{array}{ccccccc}
E & -u_{1} & & & & \\
-u_{1} & E & -u_{2} & & & \\
& -u_{2} & E & \ddots & & \\
& & \ddots & \ddots & & \\
& & & & E & -u_{N-1} \\
& & & & -u_{N-1} & E-\Sigma_{(N)}(E)
\end{array}\right)_{1,1}^{-1} .
$$

Above, the matrix $\hat{\Sigma}$ and its only non-zero element, $\Sigma_{(N)}(E)$ in the lower diagonal, is the self-energy obtained from integrating out the metallic lead. In the rest of the paper we always take $N$ to be even in order to avoid even-odd effects typical of topological systems. We also assume a smooth transition from $H_{N}$ to the metal by taking $u_{N}$ to be equal to the hopping amplitude of the metal, i.e, $u_{N}=u_{N+1}$. This gives, (see Appendix D),

$$
\Sigma_{(N)}(E)=\frac{1}{2}\left(E-i \sqrt{4 u_{N}^{2}-E^{2}}\right) \approx-i\left|u_{N}\right|,
$$

where in the last step we took the zero energy limit of the self-energy. For this case the self-energy is purely imaginary reflecting the fact that our system is open and the states decay into the metallic bulk.

We are interested in solving for $G_{S}^{(N)}(E)$, when $H_{N}$ hosts an approximate zero mode $\phi$ on the left end of the chain. Whenever the ASM $\phi$ is sufficiently strong and dominates the physics of the first site, Eq. (38) can be solved in the small $E$ limit, yielding, (see Appendix D for 
details)

$$
G_{(S)}^{(N)}=\frac{\left(\mathcal{N}_{\phi}^{(N)}+R\right)^{-1}}{i \Gamma_{A}^{(N)}+E},
$$

where we define,

$$
\begin{aligned}
& \mathcal{N}_{\phi}^{(N)}=\sum_{l=1}^{N}\left|\phi_{l}\right|^{2}, \\
& \mathcal{N}_{\eta}^{(N)}=\sum_{l=1}^{N}\left|\eta_{l}\right|^{2},
\end{aligned}
$$

and,

$$
R=\frac{\left|\phi_{N+1}\right|^{4} \mathcal{N}_{\eta}^{(N)} u_{N}^{4}}{\Sigma_{(N)}^{2} u_{1}^{2}}
$$

with

$$
\Gamma_{A}^{(N)}=\frac{\left|\phi_{N+1}\right|^{2} u_{N}^{2}}{\left|\Sigma_{(N)}\right|\left(\mathcal{N}_{\phi}^{(N)}+R\right)} .
$$

The forms of $\left|\phi_{n}\right|^{2},\left|\eta_{n}\right|^{2}$ are derived from Eq. (11), and in particular we have, for $n=2 l+1$

$$
\phi_{2 l+1}^{2}=\prod_{k=1}^{l}\left[\frac{b_{2 k-1}}{b_{2 k}}\right]^{2} .
$$

When $\phi$ is strongly localized, the quantity $R$ is vanishingly small, $R \rightarrow 0$. For this case, performing the Fouriertransform of Eq. (40), we obtain,

$$
A_{\infty}(t) \approx\left(\mathcal{N}_{\phi}^{(N)}\right)^{-1} \exp \left[-\Gamma_{A}^{(N)} t\right] .
$$

Note that, we can also numerically solve Eq. (40) on the real axis and determine the decay rate from the halfwidth of the Lorentzian of the imaginary part of the Green's function $\Im[G(E \rightarrow 0)]$. This will yield the same decay rate as in Eq. (43) when the edge mode is sufficiently long lived. Deviations from Eq. (43) will however manifest when the lifetimes become shorter. This happens when $J_{z}$ is larger, and since we are only interested in the limit of small $J_{z}$, Eq. (45), for our purposes is a good approximation for the lifetime.

Below we discuss the lifetimes for two toy models. One corresponds to an ideal SSH model coupled to leads. The other is the toy model of Eq. (15) which is an SSH model with linearly growing hopping amplitudes, and coupled to leads.

\section{A. ASM lifetime for ideal SSH model coupled to leads}

For the ideal SSH model, we take $H_{N}$ in Eq. (38) to be one where $u_{2 n-1}=u_{1}, u_{2 n}=u_{2}$. Moreover, we take the metal to have the hopping strength $u_{0}=\left(u_{1}+u_{2}\right) / 2$. The ratio of the hoppings

$$
r=\frac{u_{1}}{u_{2}},
$$

controls the topological nature of SSH model, where $r<$ 1 is topological and $r>1$ is trivial.

In the $E \rightarrow 0$ limit, the surface Green's function can be solved for exactly ${ }^{60}$ (see Appendix D for details), giving the decay rate,

$$
\Gamma_{A} \sim \frac{u_{2}^{2} r^{N}\left(1-r^{2}\right)}{u_{0}} ; \quad u_{0}=\frac{u_{1}+u_{2}}{2} .
$$

\section{B. ASM lifetime for SSH model with slope and coupled to leads}

Let us estimate the decay rate (43) for the toy model Eq. (15). Assuming the mass is a square wave of width $x_{0}$ and magnitude $M_{0}$ and using Eq. (44), the decay rate is found to be,

$$
\Gamma_{A} \propto\left|\phi_{x_{0}+1}\right|^{2}=\prod_{l=1}^{x_{0} / 2}\left[\frac{\alpha(2 l-1)+\delta-M_{0} / 2}{\alpha(2 l)+\delta+M_{0} / 2}\right]^{2} .
$$

If $\alpha \ll M_{0} \ll \delta$ and $x_{0}$ is very large we evaluate (48) by replacing sums by integrals and obtain with exponential accuracy

$$
\ln \Gamma_{A} \sim-\frac{M_{0}}{\alpha} \ln \left(\frac{\alpha x_{0}}{\delta}\right) .
$$

The above recreates the exponential dependence of the decay rate from the continuum model, Eq. (37).

\section{COMPARISON BETWEEN ED AND TOY MODELS}

In this section we compare the lifetime determined from ED, to two different estimates for the lifetime. One estimate is based on a Krylov chain of length $N$, coupled to an ideal metal, where the hoppings of the Krylov chain equal to the Lanczos coefficients in Fig. 2. The second estimate is based on the toy model Eq. (15).

We now briefly return to Fig. 1 . This figure plots $A_{\infty}$ from ED, and compares it to Eq. (45), where the latter uses the $L=12$ Gram-Schmidt orthogonalized $b_{n}$, and $N=400$. Eq. (45) accurately reconstructs the prefactor of the decay, and is also a good approximation to the decay constant. The latter is better visible in Fig. 5 . The ED lifetime shown in Fig. 5 is defined as $\Gamma^{\mathrm{ED}}=$ $1 / t^{*}$, where $t^{*}$ is the time at which $A_{\infty}\left(t^{*}\right)=\left(\mathcal{N}_{\phi}^{(N)} e\right)^{-1}$, where $\mathcal{N}_{\phi}^{(N)}$ is given in Eq. (41), with $N=400$.

The upper panel of Fig. 5 compares the ED lifetimes to the lifetimes $\Gamma_{A}^{(N)}$ determined from the Lanczos coefficients and using the formula Eq. (43). The lifetimes $\Gamma_{A}^{\text {toy }}$ 

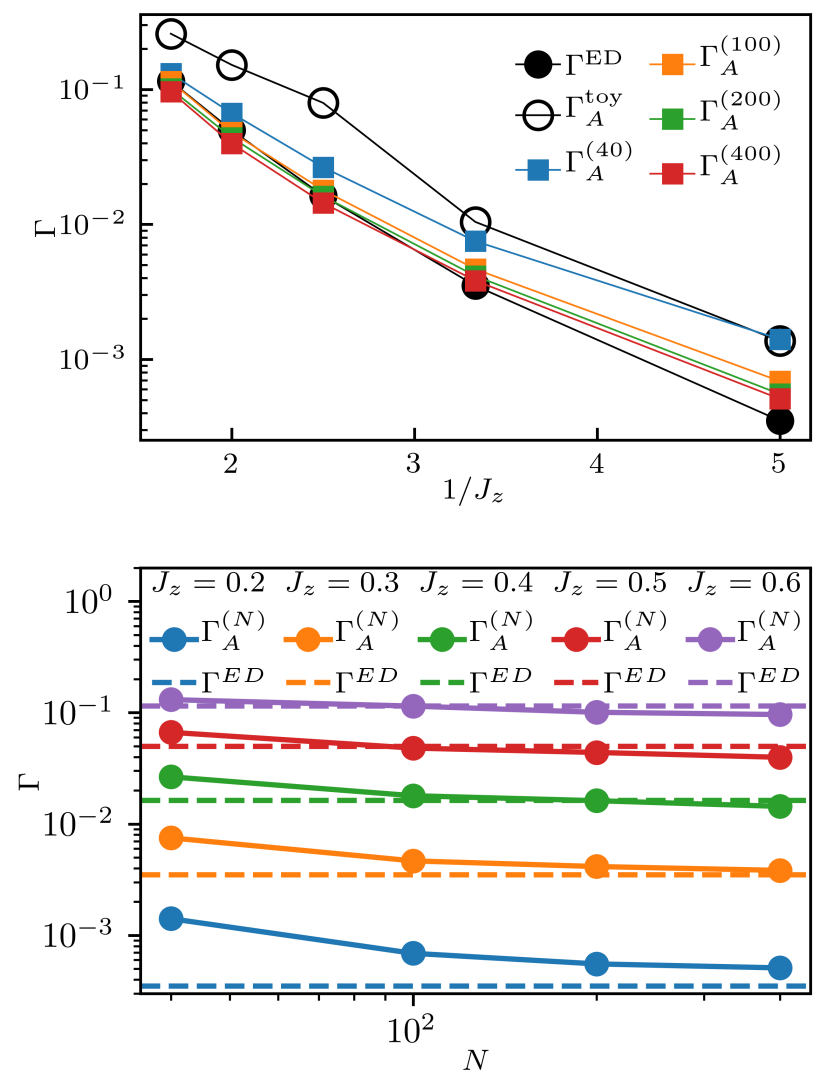

FIG. 5. Upper panel: Comparison of the decay rates $\Gamma^{\mathrm{ED}}$ obtained from ED to two approximate decay rates denoted by $\Gamma_{A}^{(N)}$ and $\Gamma_{A}^{\text {toy }}$. The ED data set is obtained from the autocorrelation function already shown in Fig. 1, with parameters $L=12, g=.3, \gamma=.9$, and $J_{z}=.2, .3, .4, .5, .6$. $\Gamma_{A}^{(N)}$ corresponds to Eq. (43) with $N=40,100,200,400$ and with the first $N$ Lanczos coefficients $b_{n}$ taken as the hopping parameters. $\Gamma_{A}^{\text {toy }}$ corresponds to Eq. (43), but uses the toy model Eq. (15), with the fitted values from Fig. 4, and taking $\beta=100$, and $N=M=50$. Lower panel: Convergence of $\Gamma_{A}^{(N)}$ to $\Gamma^{\mathrm{ED}}$, plotted against $N$ and for all $J_{z}$ values.

obtained from the toy-model are also shown, and these lifetimes are plotted against $1 / J_{z}$.

In the computation of $\Gamma_{A}^{(N)}$, a few different $N$ have been used. The dependence of $\Gamma_{A}^{(N)}$, as a function of $N$, shows how the metallic approximation performs. Increasing $N$ brings $\Gamma_{A}^{(N)}$ to within good agreement with $\Gamma^{\mathrm{ED}}$ for intermediate values of $J_{z}$. The lower panel of Fig. 5 shows the convergence of $\Gamma_{A}^{(N)}$ towards $\Gamma^{\mathrm{ED}}$ as $N$ is increased.

There are two sources of error in Eq. (43) for $\Gamma_{A}^{(N)}$. The first source of error is the negligence of contributions that are higher order in $E$ (see Appendix D). This error is manifested at larger $J_{z}$ values where, as $N$ is increased, $\Gamma_{A}^{(N)}$ fails to saturate exactly on $\Gamma^{\mathrm{ED}}$. The second source of error is the failure to reach values of $N$ that are large enough to account for the full decay of the staggering in the $b_{n}$. This source of error is visible for the smaller $J_{z}$ data-sets.

In particular, the data set for smaller $J_{z}$ shows better agreement between the lifetime obtained from ED and $\Gamma_{A}^{(N)}$ as $N$ is increased, but some discrepancy remains after setting $N$ to the largest value of $N=400$. In this case, staggering of the bulk $b_{n}$ extends far from the origin. This is also apparent in the behavior of $\left|\phi_{n}\right|^{2}$ in Fig. 2, where we see that $\left|\phi_{n}\right|^{2}$ fails to reach a minimum for the data set corresponding to smaller $J_{z}$.

We now discuss the ability of the toy model Eq. (15) to capture the essential physics. In order to make the comparison, we employ Fig. 4 where the parameters of the toy model Eq. (15) are extracted from the $b_{n}$ obtained from ED. Moreover in the toy model we set $N=M=$ $50 \gg n_{0}$, and $\beta=100$ for a sharp step. Fig. 5 shows the lifetime $\Gamma_{A}^{\text {toy }}$ which is obtained from Eq. (43) by using the parameters of the toy model. For the data sets for smaller values of $J_{z}$, the lifetime of the toy model agrees well with $\Gamma_{A}^{(N)}$, for $N=40$. For this case, both computations for the decay rate make the same metallic approximation for the bulk $b_{n}$, equivalently, both have no knowledge of any staggering present in the bulk $b_{n}$. This agreement reflects that the step profile form of the mass in the toy model is accurate for the longer lived ASM.

As previously mentioned, the deviation from the results from ED indicates that the plateau of $b_{n}$ contains staggering that contributes to the lifetime. This can be thought of as an additional region of mass, which will lead to further decay of $\left|\phi_{n}\right|^{2}$ with $n$. This additional decay, beyond the initial staggering, is the strongest and most persistent for the smallest values of $J_{z}$, as visible in Fig. 2, where even the largest $L$, do not appear to find a minima for the first $400 b_{n}$. The remaining $J_{z}$ data sets in Fig. 2, do appear to see a bottoming out for $|\phi|^{2}$, indicating that the first $400 b_{n}$ are sufficient for accounting for any staggering present in the Lanczos coefficients in the bulk.

\section{CONCLUSIONS}

In this paper we have outlined a method to calculate the non-perturbatively long lifetimes of edge modes that reside at the boundary of non-integrable spin chains. Our approach is based on using the recursion method to arrive at toy models, which can then be solved analytically. We found good agreement with the lifetimes obtained from ED and the analytic estimates from the toy models. We also have an understanding for what causes the deviation between ED and toy models. This arises primarily due to the "ideal metal" approximation made for the bulk. In fact, the smaller is $J_{z}$, the more the bulk deviates from this ideal metal limit, with staggering extending far into the bulk.

Another possible source of discrepancy between ED and the toy models is the presence of noise in the $b_{n} \mathrm{~s}$ extracted from ED. As can be readily seen from Fig. 4, 
the parameters $h_{n}$ and $\tilde{h}_{n}$ are noisy both at small $n$ where staggering is present and for large $n$ in the metallic bulk region. The fluctuations of $\tilde{h}_{n}$ at small $n$ correspond to mass fluctuations in the continuous model. As the decay rate depends on mass exponentially, the effects of this noise on the decay rate $\Gamma_{A}$ can be essential. At large $n$ the noise in $h_{n}$ results in the suppression of density of states near zero energy and might affect the tunneling from the ASM into the metallic bulk as well. The study of these effects is beyond the scope of this work.

While in this paper we presented results near the Ising limit $(\gamma \sim 1)$ of the non-integrable spin chain, our method is very general. Future studies will explore the regime away from the Ising limit, and will also apply it to Floquet $\mathrm{ASMs}^{59}$. It is also interesting to utilize this method to understand the stability of edge modes in interacting topological insulators in higher spatial dimensions.

Acknowledgements: The authors thank Anatoly Dymarsky for helpful discussions. This work was supported by the US Department of Energy, Office of Science, Basic Energy Sciences, under Award No. DE-SC0010821 (DJY and AM) and by the US National Science Foundation Grant NSF DMR-1606591 (AGA).

\section{Appendix A: Numerical methods and orthogonalization errors in the $b_{n}$}

The numerical calculation of the $b_{n}$ can be efficiently performed by representing the operators in their Paulistring basis ${ }^{47}$. However, this choice of representation does not avoid the usual costs associated with ED. In particular, for each $\left.\mid O_{n}\right)$ one needs to store the Pauli-string basis operators that constitute $\left.\mid O_{n}\right)$, and their non-zero coefficients. For non-integrable systems, one expects the number of Pauli-string basis elements in $\left.\mid O_{n}\right)$ to increase exponentially with $n$. If one works in the thermodynamic limit $^{47}$, then this exponential wall of complexity effectively caps the possible number of $b_{n}$ to roughly $n \sim 40$.

While the thermodynamic limit is ideal for studying bulk properties free of finite size effects, we choose to work with finite systems, of the same size as the ED calculations, $L \leq 14$. We are justified in studying small systems because for the parameters chosen, $A_{\infty}$ saturates for the available system sizes, as seen in Fig. 1. By considering a finite system of size $L$, our operators $\left.\mid O_{n}\right)$ are bounded in length by $2^{2 L}$, allowing us to calculate more $b_{n}$.

For large $n$, the Lanczos algorithm is susceptible to errors in producing orthogonal vectors. Eq. (7) requires only three $\left.\mid O_{n}\right)$ to be stored in memory at any given iteration step in the algorithm. As numerical error accumulates, the newly generated $\left.\mid O_{n}\right)$ will inevitably have overlap with states calculated earlier, and without stor- ing those earlier vectors, there is no way to correct for this. Typically this occurs between $n=50$ to $n=100$, which is visible in Fig. 2.

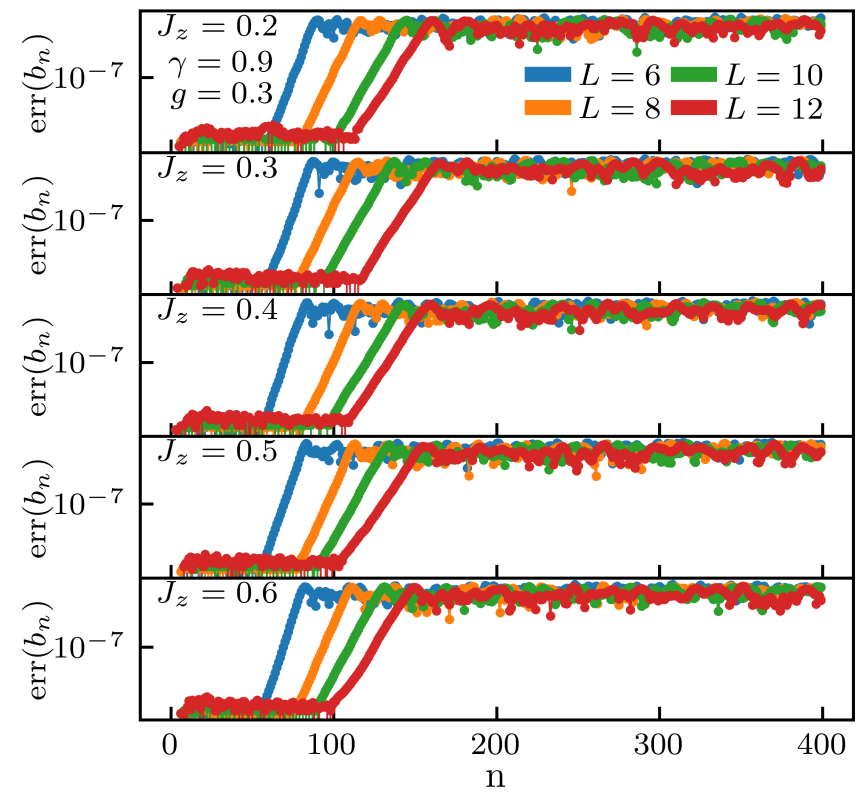

FIG. 6. Error in $b_{n}$ from performing the Lanczos algorithm without the additional Gram-Schmidt orthogonalization step, for $L=6,8,10,12$ and $J_{z}=.2, .3, .4, .5, .6$. It is clear that the onset of the error increases in $n$ as one increases $L$, and that the rate of increase in error decreases as one increases $L$. The error also remains $\approx O(1)$ at large $n$.

To correct for this orthogonalization error, one stores all $\left.\mid O_{n}\right)$ calculated, and one performs Gram-Schmidt orthogonalization on the newly generated states. Storing all calculated $\left.\mid O_{n}\right)$, for large $n$, for $L=14$, requires a large amount of memory, thus $L=12$ is the largest system size for which we perform the extra Gram-Schmidt orthogonalization step.

The differences between the $b_{n}$ obtained from the Lanczos algorithm and the full Gram-Schmidt orthogonalization are shown in Fig. 6, for the available system sizes. The error saturates for large $n$ at a value of $\mathcal{O}(1)$. Fig. 2 shows that the qualitative nature of the $b_{n}$ is unchanged, with the most noticeable feature being the reduction of the noise at large $n$ when the states are perfectly orthogonal.

\section{Appendix B: Derivation of the continuum Hamiltonian Eq. (23)}

In Eq. (19) we substitute the ansatz Eq. (20) for the wavefunction, and the ansatz Eq. (21) for the hopping amplitudes to obtain, 


$$
\begin{aligned}
& i \partial_{t}\left(\psi_{n}+(-1)^{n} \tilde{\psi}_{n}\right)=i\left[\left(h_{n}+(-1)^{n} \tilde{h}_{n}\right)\left(\psi_{n+1}-(-1)^{n} \tilde{\psi}_{n+1}\right)-\left(h_{n-1}-(-1)^{n} \tilde{h}_{n-1}\right)\left(\psi_{n-1}-(-1)^{n} \tilde{\psi}_{n-1}\right)\right] \\
& =i\left[h_{n} \psi_{n+1}-\tilde{h}_{n} \tilde{\psi}_{n+1}-h_{n-1} \psi_{n-1}-\tilde{h}_{n-1} \tilde{\psi}_{n-1}+(-1)^{n}\left(\tilde{h}_{n} \psi_{n+1}-h_{n} \tilde{\psi}_{n+1}+\tilde{h}_{n-1} \psi_{n-1}+h_{n-1} \tilde{\psi}_{n-1}\right)\right] .
\end{aligned}
$$

Since we are considering the wavefunctions and hopping parameters to be slow, while the $(-1)^{n}$ factor is rapid, we approximately solve the above by equating terms on the left and right to each other conditioned on the presence of the $(-1)^{n}$ term. This leads to the system of equations,

$$
\begin{aligned}
& i \partial_{t} \psi(n)=i\left[h_{n} \psi_{n+1}-\tilde{h}_{n} \tilde{\psi}_{n+1}-h_{n-1} \psi_{n-1}-\tilde{h}_{n-1} \tilde{\psi}_{n-1}\right], \\
& i \partial_{t} \tilde{\psi}(n)=i\left[\tilde{h}_{n} \psi_{n+1}-h_{n} \tilde{\psi}_{n+1}+\tilde{h}_{n-1} \psi_{n-1}+h_{n-1} \tilde{\psi}_{n-1}\right] .
\end{aligned}
$$

In order to take the continuum limit, we restore the lattice spacing, $n \rightarrow n a_{0}=x$, and assume long wavelengths by dropping $\mathcal{O}\left(a_{0}^{2}\right)$ and higher order terms,

$$
\begin{aligned}
& i \partial_{t} \psi(x)=i\left[2 a_{0} h(x) \partial_{x} \psi(x)-2 \tilde{h}(x) \tilde{\psi}(x)+a_{0}\left[\partial_{x} h(x)\right] \psi(x)+a_{0}\left[\partial_{x} \tilde{h}(x)\right] \tilde{\psi}(x)\right], \\
& i \partial_{t} \tilde{\psi}(x)=i\left[2 \tilde{h}(x) \psi(x)-2 a_{0} h(x) \partial_{x} \tilde{\psi}(x)-a_{0}\left[\partial_{x} \tilde{h}(x)\right] \psi(x)-a_{0}\left[\partial_{x} h(x)\right] \tilde{\psi}(x)\right] .
\end{aligned}
$$

By defining the spinor,

$$
\tilde{\Psi}=\left(\begin{array}{c}
\psi(x) \\
\tilde{\psi}(x)
\end{array}\right)
$$

and the mass,

$$
m(x)=2 \tilde{h}(x)-a_{0} \partial_{x} \tilde{h}(x),
$$

we have,

$$
i \partial_{t} \tilde{\Psi}(x)=\left[\sigma^{y} m(x)+\sigma^{z}\left\{a_{0} i \partial_{x}, h(x)\right\}\right] \tilde{\Psi}(x) .
$$

We note that the boundary condition on $\tilde{\Psi}(x)$ is, $\tilde{\Psi}(0)=$ $0=\psi(0)+\tilde{\psi}(0)$, or,

$$
\sigma^{x} \tilde{\Psi}(0)=-\tilde{\Psi}(0) .
$$

In what follows we find it convenient to set the lattice spacing $a_{0}=1$ to obtain Eq. (23) in the main text,

$$
i \partial_{t} \tilde{\Psi}(x)=\left[\sigma^{y} m(x)+\sigma^{z}\left\{i \partial_{x}, h(x)\right\}\right] \tilde{\Psi}(x) .
$$

\section{Appendix C: Lifetime in Dirac continuum model} 0

Let us start with the Dirac equation on a half-line $x \geq$

$$
\begin{aligned}
i \partial_{t} \tilde{\Psi} & =H \tilde{\Psi}, \\
H & =\sigma^{z} i \partial_{x}+\sigma^{y} m(x),
\end{aligned}
$$

where the mass is space dependent. We assume that $m(x)=m=$ const $>0$ for $0 \leq x \leq x_{0}$ and $m(x)=0$ for $x \geq x_{0}$. We also assume that the wavefunction is bounded at infinity, and that at the origin $x=0$, the boundary condition is given in Eq. (B9).
The wavefunction for $x<x_{0}$ is,

$$
\begin{aligned}
\tilde{\Psi}\left(x<x_{0}\right) & =e^{-i E t}\left[A_{1} e^{-\kappa x}\left(\begin{array}{c}
-i m \\
i \kappa+E
\end{array}\right)\right. \\
& \left.+A_{2} e^{\kappa x}\left(\begin{array}{c}
-i m \\
-i \kappa+E
\end{array}\right)\right]
\end{aligned}
$$

while the wavefunction for $x \geq x_{0}$

$$
\begin{aligned}
\tilde{\Psi}\left(x \geq x_{0}, t\right) & =e^{-i E t}\left[e^{-i E\left(x-x_{0}\right)}\left(\begin{array}{l}
1 \\
0
\end{array}\right)\right. \\
& \left.+B e^{i E\left(x-x_{0}\right)}\left(\begin{array}{l}
0 \\
1
\end{array}\right)\right] .
\end{aligned}
$$

Imposing boundary conditions at $x=x_{0}$ and $x=0$ we find the scattering amplitude $B$ to be

$$
B=-\frac{\kappa \cosh \left(\kappa x_{0}\right)-m \sinh \left(\kappa x_{0}\right)+i E \sinh \left(\kappa x_{0}\right)}{\kappa \cosh \left(\kappa x_{0}\right)-m \sinh \left(\kappa x_{0}\right)-i E \sinh \left(\kappa x_{0}\right)},
$$

where

$$
\kappa=\sqrt{m^{2}-E^{2}} \approx m-\frac{E^{2}}{2 m} .
$$

We used $|E| \ll m$ in the latter approximation. The scattering amplitude $B$ has a pole in a complex plane of $E$ at (we assume $m x_{0} \gg 1$ ):

$$
E \approx-2 i m e^{-2 m x_{0}}=-i \frac{\Gamma}{2},
$$

giving the expression for the decay rate of the quasibound state:

$$
\Gamma \approx 4 m e^{-2 m x_{0}} .
$$


Let us now note that the scattering amplitude $B$ is a pure phase $B=-e^{2 i \delta}$ with

$$
\delta=\tan ^{-1} \frac{E \sinh \left(\kappa x_{0}\right)}{m e^{-\kappa x_{0}}-\frac{E^{2}}{2 m} \cosh \left(\kappa x_{0}\right)} .
$$

We find that the derivative $d \delta / d E$ has a maximum at $E=0$ :

$$
\Delta t=\left.2 \hbar \frac{d \delta}{d E}\right|_{E=0} \approx \frac{\hbar}{m} e^{2 m x_{0}},
$$

which should be interpreted as a time delay due to scattering. As expected, the delay time (C10) is of the order as the inverse decay rate $(\mathrm{C} 8)$.

\section{Appendix D: Discrete Green's functions}

In this section we outline the steps needed to derive the results in Section V.

We first explain how the metallic bulk can be integrated out. The arguments here follow Ref. 60, but we include the steps for the convenience of the reader. We define the metallic bulk as a nearest-neighbor, tightbinding Hamiltonian $H_{B}$ with hopping strength $u_{0}$ and no onsite potential. The lattice Green's function for the metallic bulk is defined as,

$$
G_{B}=\left(E-H_{B}\right)^{-1}
$$

We will only need the first component of this matrix, denoted as the surface Green's function,

$$
G_{B}^{S}=\left(G_{B}\right)_{1,1}
$$

Using the identity,

$$
\left[\left(\begin{array}{ll}
A & B \\
C & D
\end{array}\right)^{-1}\right]_{1,1}=\left(A-B D^{-1} C\right)^{-1}
$$

we can partition $H_{B}$ as follows,

$$
\left(E-H_{B}\right)^{-1}=\left(\begin{array}{cc}
E & -u_{0} \\
-u_{0} & E-H_{B}
\end{array}\right)^{-1} .
$$

Above the $(1,1)$ element is a scalar and the lower right element is semi-infinite. Solving for the $(1,1)$ element and using Eq. (D3), we obtain,

$$
G_{B}^{S}=\left(E-u_{0}^{2} G_{B}^{S}\right)^{-1}
$$

whose solution yields,

$$
G_{B}^{S}(E)=\frac{1}{2 u_{0}^{2}}\left(E \pm i \sqrt{4 u_{0}^{2}-E^{2}}\right) .
$$

The sign above is chosen according to the initial conditions. For example, a retarded Green's function will correspond to choosing the lower sign.

If we introduce a qubit on the end of the metallic bulk $^{60}$, it will modify the Green's function as follows,

$$
(E-H)^{-1}=\left(\begin{array}{ccc}
E-v_{1} & -u_{1} & \\
-u_{1} & E-v_{2} & -u_{2} \\
& -u_{2} & E-H_{B}
\end{array}\right)^{-1}
$$

where $H_{B}$ is semi-infinite. We can solve for the inverse of the top left $2 \times 2$ matrix using Eq. (D3), giving the following inverse Green's function for the qubit,

$$
\left(\begin{array}{cc}
E-v_{1} & -u_{1} \\
-u_{1} & E-v_{2}-u_{2}^{2} G_{B}^{S}(E)
\end{array}\right)^{-1} .
$$

Due to the nearest-neighbor hopping, the effects of the bulk are contained in an energy-dependent self-energy term on the last site,

$$
\Sigma(E)=u_{2}^{2} G_{B}^{S}(E)
$$

This motivates Eq. (38) in the main text. It also explains the expression for the self-energy in Eq. (39) where the hopping $u_{2}$ between the system and the metal has been taken to be equal to the hopping in the metal $u_{0}$. Moreover the latter is denoted by $u_{N}$ in the main text.

We now plan to solve Eq. (38) in the limit of $E \rightarrow 0$. We consider $H_{N}$ to have only nearest neighbor hopping, with the hopping on site $n$ being $u_{n}$, and we exclude any onsite potential. $N$ will be taken to be even, thus $u_{N}$ will act as the coupling between $H_{N}$ and the infinite bulk. Moreover, we will treat the bulk as an ideal metal with uniform hoppings $u_{n \geq N}=u_{N}$.

We denote $F_{N}$ as the lower right most matrix element of Eq. (38), inside the inversion. By utilizing Eq. (D3), we can solve Eq. (38), by iterating backwards from $F_{N}$ all the way to $F_{1}$. Let us denote an intermediate lower right matrix element as $F_{n}$, we then have, from Eq. (D3),

$$
\begin{aligned}
F_{n-1} & =E-u_{n-1}^{2} F_{n}^{-1} \\
& =E-u_{n-1}^{2} \frac{1}{E-u_{n}^{2} F_{n+1}^{-1}} .
\end{aligned}
$$

Above, in the last line we have used the fact that the same relation holds between $F_{n}, F_{n-1}$ as between $F_{n+1}, F_{n}$. We are eventually interested in the surface Green's function $G_{(1,1)}=F_{1}^{-1}$. Below we outline the steps in the iteration.

Working to first order in $E \rightarrow 0$, and denoting $\Sigma=$ $\Sigma(E=0)$, we use that $F_{N}=E-\Sigma$. Then, iterating backwards we obtain, 


$$
\begin{aligned}
F_{N-(2 l-1)} \approx & E+\frac{u_{N-(2 l-1)}^{2} \ldots u_{N-3}^{2}}{u_{N-(2 l-2)}^{2} \cdots u_{N-2}^{2}} \frac{u_{N-1}^{2}}{\Sigma}\left[1+E\left(\frac{1}{\Sigma}\left\{\frac{u_{N-(2 l-3)}^{2} \cdots u_{N-1}^{2}}{u_{N-(2 l-2)}^{2} \cdots u_{N-2}^{2}}+\frac{u_{N-(2 l-5)}^{2} \cdots u_{N-1}^{2}}{u_{N-(2 l-4)}^{2} \cdots u_{N-2}^{2}}+\cdots+1\right\}\right.\right. \\
& \left.\left.+\frac{\Sigma}{u_{N-1}^{2}}\left\{\frac{u_{N-(2 l-4)}^{2} \cdots u_{N-2}^{2}}{u_{N-(2 l-3)}^{2} \cdots u_{N-3}^{2}}+\frac{u_{N-(2 l-6)}^{2} \cdots u_{N-2}^{2}}{u_{N-(2 l-5)}^{2} \cdots u_{N-3}^{2}}+\cdots+1\right\}\right)\right] .
\end{aligned}
$$

We now consider an $N=2 L$ subsystem $H_{N}$ and we set $l=L$ in the above obtaining,

$$
\begin{aligned}
G_{(1,1)} \approx & {\left[\left(\frac{u_{1} u_{3} \ldots u_{N-1}}{u_{2} u_{4} \ldots u_{N}}\right)^{2} \frac{u_{N}^{2}}{\Sigma}+E\left\{\left(\frac{u_{1}^{2} \ldots u_{N-1}^{2}}{u_{2}^{2} \ldots u_{N}^{2}}\right)^{2} \frac{u_{N}^{4}}{\Sigma^{2} u_{1}^{2}}\left(1+\frac{u_{2}^{2}}{u_{3}^{2}}+\cdots+\frac{u_{2}^{2} \ldots u_{N-2}^{2}}{u_{3}^{2} \ldots u_{N-1}^{2}}\right)\right.\right.} \\
& \left.\left.+\left(1+\frac{u_{1}^{2}}{u_{2}^{2}}+\cdots+\frac{u_{1}^{2} \ldots u_{N-3}^{2}}{u_{2} \ldots u_{N-2}^{2}}\right)\right\}\right]^{-1} \\
= & {\left[\left|\phi_{N+1}\right|^{2} \frac{u_{N}^{2}}{\Sigma}+E\left(\left|\phi_{N+1}\right|^{4} \frac{u_{N}^{4}}{\Sigma^{2} u_{1}^{2}} \mathcal{N}_{\eta}^{(N)}+\mathcal{N}_{\phi}^{(N)}\right)\right]^{-1} } \\
= & {\left[\left|\phi_{N+1}\right|^{2} \frac{u_{N}^{2}}{\Sigma}+E\left(R+\mathcal{N}_{\phi}^{(N)}\right)\right]^{-1}, }
\end{aligned}
$$

where we have defined the wavefunction norms, $\mathcal{N}_{\phi}^{(N)}=\sum_{l=1}^{N}\left|\phi_{l}\right|^{2}, \mathcal{N}_{\eta}^{(N)}=\sum_{l=1}^{N}\left|\eta_{l}\right|^{2}$. We have also defined $R=\left|\phi_{N+1}\right|^{4} \mathcal{N}_{\eta}^{(N)} u_{N}^{4} /\left(\Sigma^{2} u_{1}^{2}\right)$.

In its final form, the surface Green's function is,

$$
G_{(1,1)} \approx \frac{\left(\mathcal{N}_{\phi}^{(N)}+R\right)^{-1}}{\left|\phi_{N+1}\right|^{2} u_{N}^{2} \Sigma^{-1}\left(\mathcal{N}_{\phi}^{(N)}+R\right)^{-1}+E} \approx \frac{\left(\mathcal{N}_{\phi}^{(N)}\right)^{-1}}{\left|\phi_{N+1}\right|^{2} u_{N}^{2}\left(\Sigma \mathcal{N}_{\phi}^{(N)}\right)^{-1}+E},
$$

where we have set $R=0$ in the second expression, a valid approximation when $\phi$ is strongly localized. From the last expression above we can read off the lifetime as,

$$
\Gamma_{A}^{(N)} \approx \frac{\left|\phi_{N+1}\right|^{2} u_{N}^{2}}{|\Sigma| \mathcal{N}_{\phi}^{(N)}}
$$

For a SSH model with homogeneous couplings coupled to a metal, we have $u_{2 l-1}=u_{1}, u_{2 l}=u_{2}, r=u_{1} / u_{2}$, and $\left|\phi_{N+1}\right|=r^{N}$. The square of the norm for the case where the SSH part is sufficiently long is $\mathcal{N}_{\phi}^{-1} \approx 1-r^{2}$. The resulting lifetime is,

$$
\Gamma_{A}^{(N)} \approx \frac{r^{N}\left(1-r^{2}\right) u_{2}^{2}}{|\Sigma|}
$$

and is also reported in the main text.

\section{Appendix E: Independence of the lifetime on the plateau height of toy model (15)}

In this section we discuss the toy model of Eq. (15) with the goal being to explain the dependence of the lifetime on the onset of the plateau, whose position we denote by $M$. We find that the decay rate from Eq. (43), when applied to the toy model Eq. (15), is insensitive to the the position $M$ at which the plateau starts, so long as $M \gg n_{0}$, where $n_{0}$ is approximately the position at which the staggering drops to zero. This is also verified by numerically solving Eq. (38), and demonstrated in
Fig. 7.

The top panel shows two different configurations of Eq. (15) that correspond to two different values of $M$, but share the following parameters $M_{0}=1, \beta=10, n_{0}=$ $10, \alpha=.3, \delta=1$. The resulting values of $|\Im G(E)|$, from Eq. (38), are plotted in the middle panel and are in close agreement for the two different values of $M$.

The lower panel compares the time evolution on this lattice (colored dotted lines) to that of Eq. (45) (grey thick line). For the latter we use the decay-rate from the half-width of the imaginary part of the Green's function, shown in the middle panel. For the pre-factor of Eq. (45) we set $N=50>M$. The lifetime is independent of $N$, 

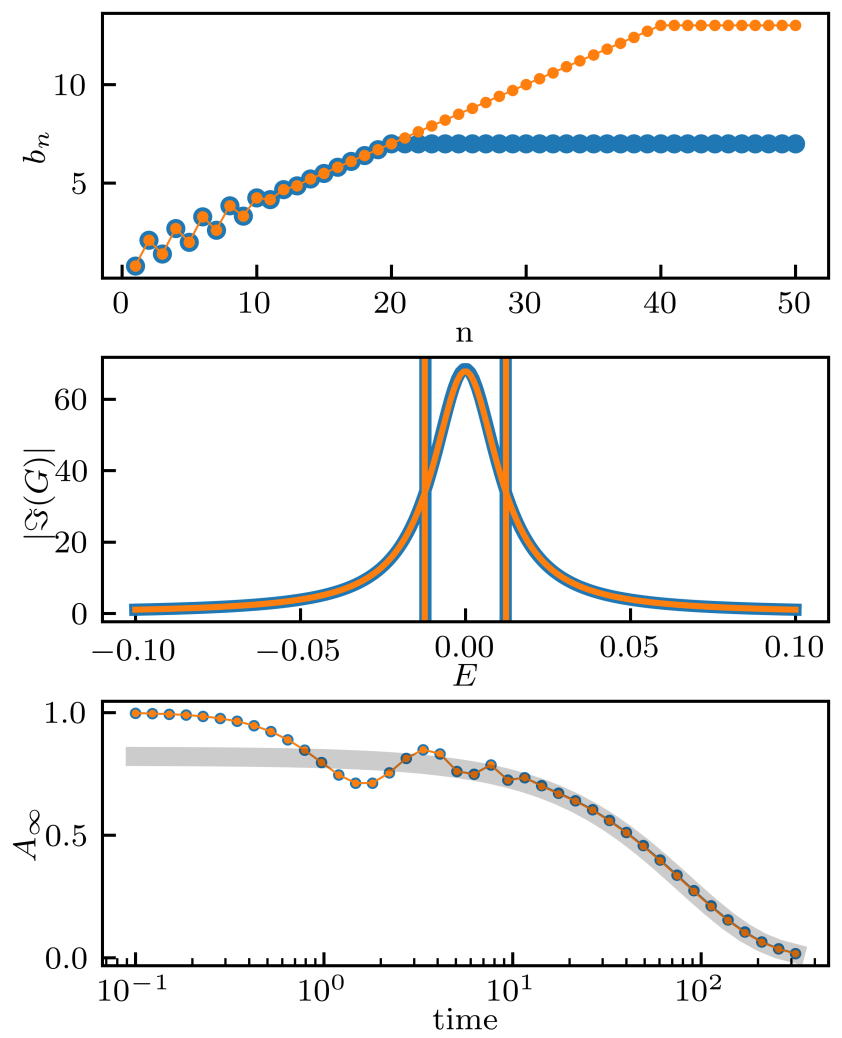

FIG. 7. Top panel shows two different configurations of the model in Eq. (15), with the same parameters save for differing positions $M=20,40$ for the onset of the plateau. The middle panel shows the imaginary part of the surface Green's function $|\Im G(E)|$ defined in Eq. (38), with $N=50$ for both $M$. The vertical lines in the middle panel mark the half-widths. In the bottom panel, the colored dotted data sets show the time evolution on the toy model lattice for both models, where the plateau is made large, but finite, extending to $1 \mathrm{e} 4$ sites. The light grey thick line shows Eq. (45), with $\Gamma_{A}^{(N)}$ corresponding to the half-widths shown in the middle panel. as long as it is greater than $M$.
In performing the numerical time-evolution (colored dotted lines in lower panel), the system is initialized with the wavefunction completely localized on the first site. The plateau is made finite, but very large in order for the autocorrelation function of the first site to be independent of the length of the plateau. The calculation is performed using iterative sparse matrix methods. ${ }^{61}$
1 C. Nayak, S. H. Simon, A. Stern, M. Freedman, and S. Das Sarma, Rev. Mod. Phys. 80, 1083 (2008).

2 J. Alicea, Reports on Progress in Physics 75, 076501 (2012).

3 C. Beenakker, Annual Review of Condensed Matter Physics 4, 113 (2013).

4 S. D. Sarma, M. Freedman, and C. Nayak, njp Quantum Information 1, 15001 (2015).

5 A. Y. Kitaev, Physics-Uspekhi 44, 131 (2001).

6 V. Mourik, K. Zuo, S. M. Frolov, S. R. Plissard, E. P. A. M. Bakkers, and L. P. Kouwenhoven, Science 336, 1003 (2012).

7 M. T. Deng, S. Vaitiekenas, E. B. Hansen, J. Danon, M. Leijnse, K. Flensberg, J. Nygård, P. Krogstrup, and C. M. Marcus, Science 354, 1557 (2016).

8 S. Nadj-Perge, I. K. Drozdov, J. Li, H. Chen, S. Jeon, J. Seo, A. H. MacDonald, B. A. Bernevig, and A. Yazdani,
Science 346, 602 (2014).

9 B. Jäck, Y. Xie, J. Li, S. Jeon, B. A. Bernevig, and A. Yazdani, Science 364, 1255 (2019).

10 C. V. Kraus, S. Diehl, P. Zoller, and M. A. Baranov, New Journal of Physics 14, 113036 (2012).

11 J. Klinovaja, P. Stano, A. Yazdani, and D. Loss, Phys. Rev. Lett. 111, 186805 (2013).

12 D. T. Liu, J. Shabani, and A. Mitra, Phys. Rev. B 97, 235114 (2018).

13 B. Bernevig and T. Hughes, Topological Insulators and Topological Superconductors (Princeton University Press, 2013).

14 M. Z. Hasan and C. L. Kane, Rev. Mod. Phys. 82, 3045 (2010).

15 S. Ryu, A. P. Schnyder, A. Furusaki, and A. W. W. Ludwig, New Journal of Physics 12, 065010 (2010).

16 X.-L. Qi and S.-C. Zhang, Rev. Mod. Phys. 83, 1057 
(2011).

17 X.-G. Wen, Rev. Mod. Phys. 89, 041004 (2017).

18 H. Katsura, D. Schuricht, and M. Takahashi, Phys. Rev. B 92, 115137 (2015).

19 D. V. Else, P. Fendley, J. Kemp, and C. Nayak, Phys. Rev. X 7, 041062 (2017).

20 J. Kemp, N. Y. Yao, C. R. Laumann, and P. Fendley, Journal of Statistical Mechanics: Theory and Experiment 2017, 063105 (2017).

21 D. E. Parker, R. Vasseur, and T. Scaffidi, Phys. Rev. Lett. 122, 240605 (2019).

22 J. Kemp, N. Y. Yao, and C. R. Laumann, arXiv:1912.05546 (2019).

23 T. Rakovszky, P. Sala, R. Verresen, M. Knap, and F. Pollmann, Phys. Rev. B 101, 125126 (2020).

${ }^{24}$ D. J. Yates, A. G. Abanov, and A. Mitra, Phys. Rev. Lett. 124, 206803 (2020).

25 T. Kitagawa, E. Berg, M. Rudner, and E. Demler, Phys. Rev. B 82, 235114 (2010).

26 M. S. Rudner, N. H. Lindner, E. Berg, and M. Levin, Phys. Rev. X 3, 031005 (2013).

27 L. Jiang, T. Kitagawa, J. Alicea, A. R. Akhmerov, D. Pekker, G. Refael, J. I. Cirac, E. Demler, M. D. Lukin, and P. Zoller, Phys. Rev. Lett. 106, 220402 (2011).

28 I.-D. Potirniche, A. C. Potter, M. Schleier-Smith, A. Vishwanath, and N. Y. Yao, Phys. Rev. Lett. 119, 123601 (2017).

29 D. J. Yates and A. Mitra, Phys. Rev. B 96, 115108 (2017).

30 D. Yates, Y. Lemonik, and A. Mitra, Phys. Rev. Lett. 121, 076802 (2018).

31 D. T. Liu, J. Shabani, and A. Mitra, Phys. Rev. B 99, 094303 (2019).

${ }^{32}$ F. Harper, R. Roy, M. S. Rudner, and S. Sondhi, Annual Review of Condensed Matter Physics 11, 345 (2020).

33 A. Lazarides, A. Das, and R. Moessner, Phys. Rev. Lett. 115, $030402(2015)$

${ }^{34}$ H. Kim, T. N. Ikeda, and D. A. Huse, Phys. Rev. E 90, 052105 (2014).

35 L. D'Alessio and M. Rigol, Phys. Rev. X 4, 041048 (2014).

${ }^{36}$ P. Ponte, A. Chandran, Z. Papić, and D. A. Abanin, Annals of Physics 353, 196 (2015).

37 A. Haldar, R. Moessner, and A. Das, Phys. Rev. B 97, 245122 (2018).

38 R. Nandkishore and D. A. Huse, Annual Review of Condensed Matter Physics 6, 15 (2015).

39 D. A. Abanin, W. De Roeck, and F. m. c. Huveneers,
Phys. Rev. Lett. 115, 256803 (2015).

40 T. Mori, T. Kuwahara, and K. Saito, Phys. Rev. Lett. 116, 120401 (2016).

41 D. A. Abanin, W. De Roeck, W. W. Ho, and F. m. c. Huveneers, Phys. Rev. B 95, 014112 (2017).

42 D. Abanin, W. De Roeck, W. W. Ho, and F. Huveneers, Communications in Mathematical Physics 354, 809 (2017).

43 P. Fendley, Journal of Statistical Mechanics: Theory and Experiment 2012, P11020 (2012).

44 A. S. Jermyn, R. S. K. Mong, J. Alicea, and P. Fendley, Phys. Rev. B 90, 165106 (2014).

45 P. Fendley, Journal of Physics A: Mathematical and Theoretical 49, 30LT01 (2016).

46 V. Vishwanath and G. Müller, The Recursion Method: Applications to Many-Body Dynamics, Springer, New York (2008).

47 D. E. Parker, X. Cao, A. Avdoshkin, T. Scaffidi, and E. Altman, Phys. Rev. X 9, 041017 (2019).

${ }^{4}$ W. P. Su, J. R. Schrieffer, and A. J. Heeger, Phys. Rev. Lett. 42, 1698 (1979).

49 W. P. Su, J. R. Schrieffer, and A. J. Heeger, Phys. Rev. B 22, 2099 (1980).

${ }^{50}$ E. Lieb, T. Schultz, and D. Mattis, Annals of Physics 16, 407 (1961).

51 T. D. Schultz, D. C. Mattis, and E. H. Lieb, Rev. Mod. Phys. 36, 856 (1964).

52 S. Sachdev, Quantum Phase Transitions, 2nd ed. (Cambridge University Press, 2011).

53 A. Altland and M. R. Zirnbauer, Phys. Rev. B 55, 1142 (1997).

54 L. Fidkowski and A. Kitaev, Phys. Rev. B 83, 075103 (2011).

55 L. Fidkowski, R. M. Lutchyn, C. Nayak, and M. P. A. Fisher, Phys. Rev. B 84, 195436 (2011).

56 A. Dymarsky and A. Gorsky, Phys. Rev. B 102, 085137 (2020).

57 J. L. F. Barbón, E. Rabinovici, R. Shir, and R. Sinha, Journal of High Energy Physics 2019, 264 (2019).

58 A. Avdoshkin and A. Dymarsky, arXiv:1911.09672 (2019).

59 D. J. Yates, F. H. L. Essler, and A. Mitra, Phys. Rev. B 99, 205419 (2019).

60 M. Zaimi, C. Boudreault, N. Baspin, H. Eleuch, R. MacKenzie, and M. Hilke, (2019), arXiv:1910.09926.

61 "Krylovkit.jl," https://github.com/Jutho/KrylovKit. jl. 